\title{
Next-Generation Tactical-Situation-Assessment Technology (TSAT): Chat
}

\author{
Emily W. Medina ${ }^{1}$, Sunny Fugate ${ }^{2}$, LorRaine Duffy ${ }^{3}$, Dennis Magsombol ${ }^{4}$, \\ Omar Amezcua ${ }^{5}$, Gary Rogers ${ }^{6}$, and Marion G. Ceruti ${ }^{7}$ \\ Space and Naval Warfare Systems Center, San Diego (SSCSD), \\ ${ }^{1}$ Code $2734,{ }^{2}$ Code $2725,{ }^{3}$ Code $246207,4,5,6$ Code $246204,{ }^{7}$ Code 246206 \\ 53560 Hull Street, San Diego, CA 92152-5001 \\ ewilsonespawar.navy.mil, fugateespawar.navy.mil, \\ lorraine.duffy@navy.mil, dennis.magsombol@navy.mil, \\ omar.amezcua@navy.mil, gary.rogers@navy.mil, \\ marion.ceruti@navy.mil
}

\begin{abstract}
This paper presents concepts, content, status, applications and challenges of chat as used in the military context of secure net-centric command and control. It describes the importance of chat as it contributes to situation assessment and the common operating picture, which presents current collective knowledge of the battle space. The paper discusses future chat capabilities and outlines the road ahead for the TSAT project.
\end{abstract}

Keywords: Chat, collaborative applications, Common Operational Picture, network-content management, social-network applications.

\section{Introduction}

Chat is an important tool commonly used on the Web. Chat also is one of the many tools, including web services that enable net-centric military operations. Chat for netcentric communications in defense-related situation assessment is one of the primary activities that drive command and control $\left(\mathrm{C}^{2}\right)$ or battle-space management. The Department of Defense uses mostly Internet-Relay Chat (IRC), which is an internetbased technology. The TSAT research-and-development project is focused on improving chat using new technology. Chat's value and maximum benefit can be realized only with the context in which it was developed.

In distributed $\mathrm{C}^{2}$, the primary tool for consistency in operational situation assessment is the Common Operational Picture (COP) and the daily briefings and updates that define relevant objects, their relationships, their intent and the commander's response to the situation or threat. The COP is geographically oriented, distributed, shared space for maintaining and presenting the current status and collective knowledge of the battle space. The tactical war fighter usually provides the context for situation assessment, the maintenance of which depends on moment-to-moment, daily, and long-term updates of the variables of interest and the time evolution of these variables. The primary method for capturing this information resides in voice and written communications, with an increasing reliance on tactical-text chat [3], [9]. 
At the moment, opportunities to improve its policy or practice seem remote, given the current political and economic outlook.

In this same vein, transfer of understanding relies heavily on improper, unwieldy, and low-information content methods of communicating context: the written word in text chat; Defense Message System (DMS) communications; or embedded within typesetting and presentation software such as Microsoft Word and Powerpoint ${ }^{\circledR}$ documents. It is alarming that our daily tactical communication updates occur through the use of high-overhead, low information density, non-interoperable, or proprietary information formats such as archaic message formats, document typesetting software, or bullet-list presentation software, The simplest of these text formats generally require the isolation of simplistic and jargon heavy content and the complete disposal of relevant context [11]. Whereas these technologies have valid uses: the broadcast of official and formal announcements; the publication of documents; or the presentation of simple concepts to an audience, they will never be suitable medium for tactical communications.

The operational Navy has an urgent requirement for better organization of the large amount of electronic information. Unorganized and content-heavy information, like that found in United States Message Text Format (USMTF) messages, chat room logs, or websites, is altogether too much for one user to assimilate, compare, analyze and use to initiate an action in a timely manner. A capability needs to be developed to support information transfer in the form of written context in situation assessment. New technology provides better ways to transfer information rapidly than the inefficient text chat and DMS paragraph or text document formats obscured by inane typesetting information and features.

We can improve the content and methods of delivery of collaborative communications for command and control in a network-centric environment. Chat is an important part of the technical approach to improve the efficiency of knowledge sharing through communication of situation-assessment context. The importance of chat is evident considering that the content of chat messages drives real-time targeting and battle-space management [9]. This content can be enhanced through linguistic research and analysis, thus improving methods for determining chat context themes. Linguistic research also can help develop a novel approach to acquiring the situational assessment themes of chat content from their human writers by building a prototype "chatbot."

\section{Chat Status}

Today's chat is a technology for sharing context. Chat is the primary and often the only means of communicating situation updates on intermittent and discontinuous networks, superseding radio communications with text-only descriptions. For example,

(1) Strike-group Concept of Operations (CONOPS) establishes 500 to 800 chat rooms with between 2,000 and 4,000 users in each "channel" based on functional roles.

(2) Joint CONOPS establishes hundreds of chat rooms with joint-service participation based on mission objectives. It enables joint access to service-specific chat rooms to maintain non-intrusive situation assessment of service-specific activities [9]. 
Chat functions include support of the following activities:

(1) Real-time targeting;

(2) Edge users with limitations of low and/or intermittent bandwidth;

(3) Immediate updates to COP context;

(4) General information sharing of updates on a regular basis continuing for months to establish the management of operational tempo and battle rhythm;

(5) Cross-domain operations.

An example has been observed of Mid-East dynamic target relocation based on chat interchange between a pilot and a ground-reconnaissance team. This is not an isolated or unusual case.

\section{Chat Development Benefits, Issues, and Challenges}

Fleet usage of chat is widespread for current operations but vastly inadequate for information retrieval and management. As important as it is, chat is too text driven, slow, and ambiguous. For these reasons, chat cannot provide the best accommodations to edge (tactical) users operating in extreme and hostile environments, such as:

(1) Chemical-biological war fighters in protective suits called "MOPP gear;"

(2) Special operations personnel in hostile situations;

(3) Clean-up crews and search-and-rescue (SAR) personnel in oil spills, fires, earthquakes, and tsunamis;

(4) Extreme-edge users on minesweepers and in submarines;

(5) Other users in oxygen-deprived or toxic-atmospheric environments.

Table 1. Benefits and issues of TSAT chat technology development

\begin{tabular}{|c|c|c|}
\hline Technology & Properties of each medium & Technology shortfalls \\
\hline $\mathrm{COP}$ & $\begin{array}{l}\text { - Comprehensive } \\
\text { - Visually integrative }\end{array}$ & $\begin{array}{l}\text { - Unavailable in field } \\
\text { - } \quad \text { Poorly communicable }\end{array}$ \\
\hline Text & $\begin{array}{l}\text { - } \text { Persistent } \\
\text { - Immediate } \\
\text { - Easy to implement \& use } \\
\text { - Expressive } \\
\text { - Prevalent operational acceptance }\end{array}$ & $\begin{array}{l}\text { - } \\
\text { - } \\
\text { - } \\
\text { - Amoor field interface } \\
\text { - Slow commul or symbol integration } \\
\text { - } \\
\text { Not stealthy }\end{array}$ \\
\hline Voice \& Video & $\begin{array}{l}\text { - } \text { Many solved problems } \\
\text { - Immediate } \\
\text { - Ostensive/direct } \\
\text { - Intuitive interfaces } \\
\text { - Mature technology } \\
\text { - Video is gesture capable }\end{array}$ & 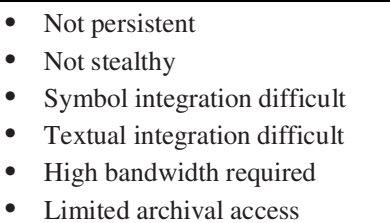 \\
\hline
\end{tabular}

Observation and statistical analysis of trends in chat-room text constitute a subset of the larger overall issue of identifying and managing large collections of unstructured text. The Internet and email are prime examples of such collections. Chat entries hold vast amounts of information that easily can be misconstrued or forgotten 
altogether [3]. Therefore, chat-room text itself must be scrutinized and the domain well defined before any further improvements on military chat commence. Issues and benefits that relate to secure net-centric chat are summarized in Table 1, showing how development of augmented chat capabilities can resolve outstanding technology shortfalls for COP, text, and through multi-modal tactical applications, voice and video.

Chat users need to integrate their information with other information sources at their disposal: geographic land-based terrain maps, the COP, and non-geographic computer-based "terrain" (e.g. global network operations and network topology). They also must be able to incorporate object relationships and time into a precise context that can be fused with other data to present an accurate COP and situation assessment, to serve as a basis for current command decisions, and for later study in operations analysis.Multiple criteria need to be established to institute rigor in chatroom text analysis. Metrics must be identified and measured against one another, and even compared with existing benchmark methods for chat analysis that are used in academia [1], [2], [4], [5], [6], [7], [10]. A few methods initially seem promising. For example, a user might be observed in a real-time environment to measure the amount of time taken to process a message and disseminate its contents to involved parties. Alternately, a user could be observed in attempts to locate topical information in a chat database. Another method might involve comparing the time a user takes to process a message with English text to the amount of time the user takes if visual symbols are substituted for text.

\section{Chat Content}

At the onset of Operations Iraqi Freedom and Enduring Freedom, the joint tactical community resorted to Internet Relay Chat (IRC) to transmit contextual information. This represents a late 1980s Internet capability. IRC has seen little or no improvement in the past two decades [3], [9]. With chat becoming more widespread in operational and tactical communities, the need is growing to understand the following.

(1) The content that is communicated in chat sequences for situation updates;

(2) How to categorize and parse the information more efficiently to various other repositories, such as databases; and

(3) How to communicate quickly the nuances of evolving situations to distributed forces in an improved (and more automated) format.

Concomitantly, knowledge of context resides first in the warfighter's mind. Today, this knowledge is communicated in gestures that are outside of computer networks and, therefore, are not stored in digital format. The present work includes the intent to acquire that contextual information using conversational query (in the guise of a "chatbot") that constitutes an interesting and novel approach to information gathering. Traditional data-acquisition technologies have focused on data storage sites, whereas a key focus of the present work in TSAT is in the human network of incidental information, or "color commentary" that often is disregarded and rarely is preserved with other historical accounts unless one can recall and record a personal conversation with the information source. Whereas few metrics exist for chat-room 
text analysis, to improve the current situation with chat, a statistical analysis of chat structure, topical organization, and user trends first must be performed.

\section{Future Chat Capabilities}

The goals of unstructured text chat research are twofold. First, linguistic analysis must discover statistically the topics that are most likely to reoccur in chat rooms. After these themes are identified, further study will focus on the best means of managing large databases of chat text. A few approaches have gained popularity when observing chat-room data, among them topic thread detection, and user-thread detection [1], [5], [6], [8], [10]. Topic thread detection would aid a user in identifying important entries, whereas user-thread detection would facilitate error detection and the identification of anomalous behavior. In the near term, metrics will be identified, tested, and evaluated. Thus, several near-term efforts need to be accomplished to advance chat beyond its current state, including short-term goals such as:

(1) Select a chat domain as the focus the study.

(2) Access secure-net-centric chat for content analysis.

(3) Obtain a statistically significant data set.

(4) Identify and test chat metrics.

(5) Perform statistical analysis of chat-room topical content, user trends, and battle-field themes.

(6) Construct a prototype for a chat-user database

(7) Construct a prototype for topic-thread detection.

In ten years, chat will augment text with an Icon/Visual/Symbolic-based language. This language will be easy to understand, visual, international, and capable of depicting geographic and non-geographic, network-based battlefields. Future chat will use very low bandwidth ( $<300 \mathrm{bps})$ and operate robustly in conditions of intermittent connectivity. The chat of the future will allow the user to portray complex concepts more efficiently than that of today. These complex concepts will be linguistically derived from then-current chat content to discipline language development. Tomorrow's chat will be keyboard independent because war fighters will use it for chemical and biological defense in extremely contaminated environments and in any other place where typing on a keyboard is not an option. For example, environmentally challenged astronauts will use it in space. Future rescue crews will use future chat in toxic, fire, or oxygen-deprived environments. Special Forces require stealth and immediate, persistent, line-of-sight independent communication tools, ease of use, and comprehensive situation updates from a COP in minimum time with minimum effort. This technology will provide key capabilities for special-operations personnel who must maintain a low probability of communication interception and detection in very noisy or stealth environments. The success of chat is tied to other technological advances. For example, future improvements to graphic user interface, textual representation, and method of use rely solely on an accurate portrayal of the military-chat domain. 


\section{The Road Ahead for the TSAT Project}

Research goals include the following.

(1) Determine metrics for ease of use, efficiency, and ambiguity resolution.

(2) Answer the following key research question: "Does TSAT net-centric chat increase information capacity or communications efficiency?"

(3) Linguistically analyze current chat content for linguistic "themes."

(4) Build a prototype candidate language.

(5) Integrate linguistic themes with visual language prototype (new symbols).

(6) Introduce new symbols and visual language elements into chat.

(7) Perform "Visual Language" experiments testing human factors.

(8) Achieve a technology transition in a long-range program in linguistic analysis technology for tactical communications.

(9) Incorporate some Web-based technologies into chat.

\section{Summary}

The objective of the TSAT project is to improve the contextual information interface that accompanies traditional situation assessment. The program focus is to revolutionize the tactical text-chat interface including content, technique, application, network management, and hardware interface. Another important goal is to improve the transfer of contextual information to the COP and among distributed operational and tactical war fighters, regardless of the specific context. When completed, this research and development project will offer a significant improvement in the ability of war fighters at all echelons to share knowledge rapidly and accurately. Not only will it improve the communication of current contextual information, it will extend the range of capabilities both in terms of how information is exchanged.

\section{Acknowledgements}

The authors thank the Defense Threat Reduction Agency and the SSCSD Science and Technology Initiative for their support of this work. This paper is the work of U.S. Government employees performed in the course of employment and no copyright subsists therein. It is approved for public release with an unlimited distribution.

\section{References}

1. Bengel, J., Gaulch, S., Mittur, E., Vijayaraghavan, R.: ChatTrack: Chat room topic detection using classification (2004) http://citeseer.ist.psu.edu/bengel04chattrack.html

2. Elnahrawy, E.: Log-based chat room monitoring using text categorization: A comparative study. In: Proceedings of the International Association of Science and Technology for Development Conference on Information and Knowledge Sharing (IKS) (November 2002) 
3. Eovito, B.: An assessment of Joint chat requirements from current usage patterns. Thesis, Naval Postgraduate School (NPS) (June 2006)

4. Hearst, M.: Multi-paragraph segmentation of expository text. In: Proceedings of the 32nd Annual Meeting of the Association for Computational Linguistics (June 1994)

5. Kolenda, T., Hansen, L.K., Larsen, J.: Signal detection using ICA: Application to chat room topic spotting (2001) http://citeseer.ist.psu.edu/kolenda01 signal.html

6. Lin, C.Y.: Knowledge-based automatic topic identification. In: Proceedings of the Association for Computational Linguistics (ACL) (June 1995)

7. Salton, G., Singhal, A., Buckley, C., Mitra, M.: Automatic text decomposition using text segments and text themes. In: Proceedings of the 7th ACM Conference on Hypertext (March 1996)

8. Schmidt, A.P., Stone, T.K.M.: Detection of topic change in IRC chat logs. http://www.trevorstone.org/school/ircsegmentation.pdf

9. Simpson Jr, M.L.: A user's epistle on TextChat tool acquisition. In: Proc. of the Command and Control Research and Technology Symposium (CCRTS 2006) (June 2006)

10. Wayne, C.L.: Topic detection and tracking in English and Chinese. In: proc. of the 5th International Workshop on Information Retrieval with Asian Languages (IRAL) (September-October 2000)

11. Tufte, E.: Beautiful Evidence. Graphics Press (July 2006) 